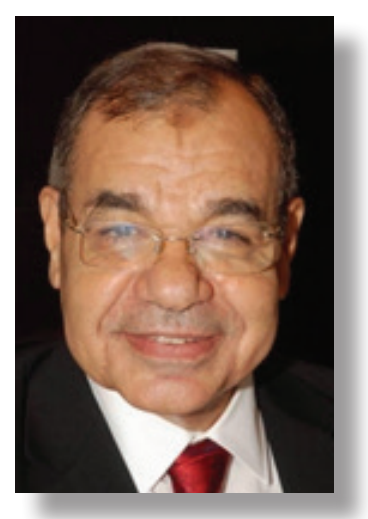

\title{
Use of ICT To Confront COVID-19
}

\author{
Yousry S. Elgamal \\ D.Sc.* \\ Former Minister of Education, Egypt \\ Prof. of Computer Engineering \\ Chairman of Computer Scientific Society \\ Senior Advisor, AASTMT \\ Chairman, Information \& Communication Committee at the National Committee of \\ Education, Culture, and Science [UNESCO, ALECSO, ISESCO)
}

The impact of COVID-19 outbreak has now become of grave concern for virtually every country in the world, and forced a majority of countries into partial or complete lockdowns. World Health Organization "WHO" reports show more than 150 Million confirmed cases, with a death toll exceeding 3 Million worldwide as of 28 April $202{ }^{[1]}$. The pandemic highlighted the crucial role played by Information and Communication Technology in keeping businesses running and societies functional in times of lockdowns and quarantines ${ }^{(2)}$. COVID-19 has accelerated our dependence on digital technologies and highlighted the life-saving benefits of connectivity. Some of the most important technologies and applications used to combat the Virus and mitigate its impacts are discussed in this article ${ }^{[3]}$.

\section{Internet of Things [loT]:}

In the health care and medical services arena, Applications of loT include: monitoring patients from a remote location, tracking medication orders, and using wearables to transmit health information to the concerned health care professionals. Several innovators, medical organizations, and government bodies are looking to utilize loT tools in order to reduce the burden on the health care systems $^{[4]}$.

Internet-connected thermometers, known as Smart Thermometers are used to screen people for high temperature. These thermometers are linked to a mobile application, which allows them to transmit their readings to the medical center immediately. Once received, this data is assimilated to generate daily maps showing which regions are witnessing an increase in high fevers, thereby allowing authorities to identify potential hotspots. ${ }^{\text {(5) }}$

Telehealth is the practice of using loT to facilitate remote patient monitoring, thus; allowing clinicians to evaluate, diagnose, and treat patients without needing any physical interaction with them. Adopting telehealth techniques lessened the burden on the overworked hospital staff, and has reduced the risk of spread of the virus from the infected individuals to the healthcare personnel. Several telemedicine tools were developed lately including Chatbots that can make initial diagnosis based on symptoms identified by patients, teleconsultation software, and portable medical tablets. However, the true potential of telemedicine can only be realized when existing telemedicine platforms are used in conjunction with other technologies such as drones, robots, smart wearables, and next-generation $5 G$ cellular networks. China developed a smart phone app health rating system that is tracking millions of people daily. It assigns three colors to people (green, yellow, and red] on the basis of their travel and medical history. Only those who were assigned a green color could be allowed in public spheres ${ }^{\left[{ }^{6}\right]}$. 
Robotics:

Robots were on the front line to prevent the spread of Corona virus. They served to spray disinfectants, prepare meals at hospitals, double up as waiters in restaurants, dispense hand synthesizers, and deliver food to those in quarantine. In many hospitals, robots were also performing diagnosis, conducting thermal imaging, and transporting medical samples ${ }^{[7]}$. A hospital at Wuhan was being staffed entirely by robots. Most of the devices are loT enabled and services are carried out by robots. The initial screening of the patients is done by $5 \mathrm{G}$ enabled thermometers that send instant updates. There are rings and bracelets that are connected to the Al platform so that it can monitor all changes in the body ${ }^{(8)}$. In addition, it is suggested that a kind of ambulance robot, operated remotely and supplied with medical tools such as a smart thermometer, automatic external defibrillator, corona virus test kit, and other instruments, could be used to instruct the public on appropriate actions to take in public spaces in an emergency ${ }^{(9)}$. In hotels and restaurants, robots could carry luggage and guide guests through front desk services. Miscellaneous tasks such as cleaning rooms and pouring coffee were also done by robots. Robots, equipped with $\mathrm{Al}$, can potentially provide information, do housekeeping work, provide food services, and help to comfort and entertain the customer in a safe way. Restaurants have also adopted robots to transfer goods from warehouses to trucks and customers amid the COVID-19 pandemic to limit potential virus contamination. At the time of risk of people-to-people contact, Autonomous Vehicles are proving to be of great utility in delivering essential goods like medicines and food items, and disinfecting hospitals. Electric street cleaning vehicles were also used at times of lockdown.

Drone Technology:

Drones can provide numerous benefits in managing the COVID-19 pandemic due to the minimized human interaction and the ability to reach otherwise inaccessible areas. Several countries around the world have joined forces with numerous researchers and innovators in an attempt to find ingenious ways of using drones to fight the COVID-19. Drones were transporting both medical equipment and patient samples, saving time and enhancing the speed of deliveries, while preventing contamination of medical samples ${ }^{(10)}$. Agricultural drones were spraying disinfectants in the countryside. China employed the use of drones equipped with infrared cameras to carry out large-scale temperature measurements in several residential areas. Drones powered with facial recognition were also being used to broadcast warnings to the citizens not to step out of their homes, and chide them for not wearing face masks ${ }^{[11]}$. In India, a "corona combat" drone, is equipped with a thermal camera for screening individuals, a night vision camera for monitoring the crowd, a portable medical box for carrying essential medical supplies, a loudspeaker for making announcements, and a disinfectant tank with a capacity of 10 liters for sanitizing public spaces.

Artificial Intelligence:

With the help of data analytics and predictive models, Al proved to be a highly effective tool against the COVID-19 Pandemic ${ }^{[12]}$. Risk prediction is one of the important Al applications during the Pandemic. The risk of getting infected is a function of numerous factors where mathematical modeling would not yield fruitful results. However, a comprehensive analysis of these factors integrated with Al techniques, can offer a more precise and reliable prevision of individual risk profiles ${ }^{[13]}$. Once a person is infected, Al capabilities can also be used to determine the probability of survival and the requirement of ICU treatment for COVID-19 patients. Al techniques, particularly machine learning algorithms, can also be used to correlate the patient's data parameters with a specific drug's 
usage. In the medical diagnosis and screening arena, Al enhanced the tools of face scanners, medical imaging, and voice detection systems for rapid diagnosis.

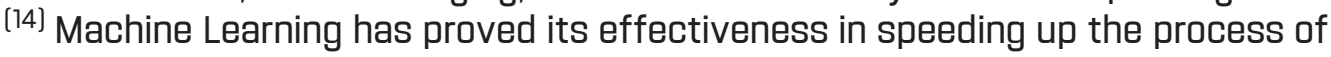
drug development. Scientists are also using Al to help identifying existing drugs that can be beneficial in treating the COVID-19. For Virus modeling and analysis, Machine Learning happens to be one of the most effective tools. ML models can be used to compare the viral genome with known genomes and identify existing similarities $^{(15)}$. Other applications of $\mathrm{Al}$ include curbing the propagation of fake news and misinformation of social media platforms as well as enforcing the lockdown measures.

\section{$5 G$ Technology:}

In comparison to $4 \mathrm{G}, 5 \mathrm{G}$ is expected to have better performance in terms of higher speed, lower latency, wider range, increased availability, and more reliability. Together with other technologies like loT and Al, $5 \mathrm{G}$ technology has the potential to revolutionize the healthcare sector ${ }^{[16]}$. $5 \mathrm{G}$ can enable immersive virtual and augmented reality [VR/AR] applications, which can lead to an interactive experience in telemedicine, and equip medical teams to provide immediate expertise in regards to possible complications and treatment strategies. In China, 5G-enabled medical imaging platforms allowed for real-time diagnosis of COVID-19 patients, and in doing so, relieved some of the load on the hospital's medical staff. A 56 Infra-Red thermal imaging monitoring system can enable the realtime temperature of moving bodies with high accuracy and precision. The data accumulated by the systems can then be transmitted to the central monitoring system with ultra-low latency using $5 G$ networks ${ }^{[17]}$.

\section{Conclusion:}

COVID-19 has demonstrated the importance of digital readiness to allow life to continue -as much as possible- during pandemics. Supporting a digitized world and staying current in the latest technology will be essential for any country to remain competitive in a post COVID-19 world. Digitization and pandemics have accelerated changes to jobs available to humans. The pandemic has highlighted and exacerbated inequalities of all kinds, including the digital divide. Those without access to digital technology - almost half of the world - are denied opportunities to study, communicate, trade, work, and participate in much of what is now normal life for the richer half of the world.

\section{References:}

1. World Health Organization. "Coronavirus disease 2019 (COVID-19): Situation report, " [2021].

2. Chamola, Vinay, et al. "A Comprehensive Review of the COVID-19 Pandemic and the Role of loT, Drones, Al, Blockchain, and $5 \mathrm{G}$ in Managing its Impact." IEEE Access 8[2020]: 90225-90265.

3. D. S. W. Ting, L. Carin, V. Dzau, and T. Y. Wong, "Digital technology and COVID-19," Nature Med., vol. 26, no. 4, pp. 459-461, Apr. 2020.

4. M. Rouse. What is loMT (Internet of Medical Things) or Healthcare loT?Definition From Whatls.com. IoT Agenda, Aug. 2015. [Online]. Available: https://internetofthingsagenda.techtarget.com/definition/loMT-Internet\%of-Medical-Things.

5. J. J. P. C. Rodrigues, D. B. D. R. Segundo, H. A. Junqueira, M. H. Sabino, R. M. Prince, J. Al-Muhtadi, and V. Hugo C. De Albuquerque, "Enabling technologies for the Internet of health things," IEEE Access, vol. 6, pp. 13129-13141, 2018. 
6. T. Hornyak. What America Can Learn From China's Use of Robots and Telemedicine to Combat the Coronavirus. CNBC. Mar. 2020. [Online]. Available: https://www.cnbc.com/2020/03/18/how-china-isusing-robotsand-telemedic\%ine-to-combat-the-coronavirus.html.

7. Zeng, Zhanjing, Po-Ju Chen, and Alan A. Lew. "From high-touch to high-tech: COVID-19 drives robotics adoption." Tourism Geographies [2020): 1-11.

8. G.-Z. Yang, B. J. Nelson, R. R. Murphy, H. Choset, H. Christensen, S. H. Collins, P. Dario, K. Goldberg, K. Ikuta, and N. Jacobstein, "Combating COVID-19-The role of robotics in managing public health and infectious diseases," Sci. Robot., vol. 5, no. 40, Mar. 2020, Art. no. eabb5589, doi: 10.1126/scirobotics. abb5589.

9. B. Marr. Robots And Drones Are Now Used To Fight COVID-19. Forbes, Mar. 2020. [Online].

10. M. Sharma, "How drones are being used to combat COVID19," Geospatial World, Apr. 2020. [Online].

11. J. Yang, 3 Ways China is Using Drones to Fight Coronavirus. Beijing, China: World Economic Forum, Mar. 2020. Accessed: Apr. 30, 2020. [Online].

12. Latif, Siddique, et al. "Leveraging Data Science To Combat COVID-19: A Comprehensive Review."

13. K. A. Wittbold, C. Carroll, M. Iansiti, H. M. Zhang, and A. B. Landman, "How hospitals are using Al to battle Covid-19," Harvard Business Review, Apr. 2020. [Online].

14. W. Naude, "Artificial intelligence against covid-19: An early review," Medium, Apr. 2020. [Online].

15. X. Jiang, M. Coffee, A. Bari, J. Wang, X. Jiang, J. Huang, J. Shi, J. Dai, J. Cai, T. Zhang, Z. Wu, G. He, and Y. Huang, "Towards an artificial intelligence framework for data-driven prediction of coronavirus clinical severity," Comput., Mater. Continua, vol. 62, no. 3, pp. 537-551, 2020.[Online].

16. Q. Xiaoxia, "How emerging technologies helped tackle COVID-19 in China," World Economic Forum, Apr. 2020. [Online].

17. D. $\mathrm{Li}$, " $5 \mathrm{G}$ and intelligence medicine-How the next generation of wireless technology will reconstruct healthcare?" Precis. Clin. Med., vol. 2, no. 4, pp. 205-208, Dec. 2019

Prof. Yousry Elgamal, Professor of Computer Engineering, senior advisor at The Arab Academy for Science and Technology, and Chairman of The Computer Scientific Society [CSS], Alexandria-Egypt. He served as The Minister of Education of Egypt 2005-2010, Chairman, Board of Trustees, Egypt Japan University of Science and Technology [E-JUST] 2010-2014, and the senior consultant of the National Telecommunications Institute of Egypt. He is a member of the group of experts preparing The Global Knowledge Index, and The Chairman of The Information and Communication Committee at The National Committee of Education, Culture, and Science [UNESCO, ALECSO, ISESCO]

Elgamal has also served in a number of capacities at The Arab Academy for Science and Technology and Maritime Transport including Vice-President for Education and Research, Founding Dean of College of Engineering and Technology, Founding Chairman of Electronics and Communication Department, and Assistant to the President for Informatics. He served also as a Lecture of Nuclear Electronics at The Atomic Energy Agency [IAEA].

He received his B.Sc. in Electrical Engineering from the University of Alexandria 1968, his M.Sc. form Ain-Shams University 1977. Prof. Elgamal holds a D.Sc. in Computer Science from the George Washington University 1985, and a recipient of Richard Merwin Award 1984. He is a board member of the Center of Special studies and Programs [CSSP] and the Center of Science and Planetarium, Bibliotheca Alexandrina. Dr. Elgamal is an active member of the Civil Society of Alexandria as a chairman of The Society of Friends of Music and Arts, and former president of The Yacht Club of Egypt in Alexandria. 